21st Particles and Nuclei International Conference (PANIC 2017)

International Journal of Modern Physics: Conference Series

Vol. 46 (2018) 1860045 (6 pages)

(C) The Author(s)

DOI: $10.1142 / \mathrm{S} 2010194518600455$

\title{
Observational Role of Dark Matter in $f(R)$ Models for Structure Formation
}

\author{
Murli Manohar Verma \\ Department of Physics, University of Lucknow, Lucknow, 22600\%, India \\ sunilmmv@yahoo.com \\ Bal Krishna Yadav \\ Department of Physics, University of Lucknow, Lucknow, 226007, India \\ balkrishnalko@gmail.com
}

Published 3 May 2018

\begin{abstract}
The fixed points for the dynamical system in the phase space have been calculated with dark matter in the $f(R)$ gravity models. The stability conditions of these fixed points are obtained in the ongoing accelerated phase of the universe, and the values of the Hubble parameter and Ricci scalar are obtained for various evolutionary stages of the universe. We present a range of some modifications of general relativistic action consistent with the $\Lambda \mathrm{CDM}$ model. We elaborate upon the fact that the upcoming cosmological observations would further constrain the bounds on the possible forms of $f(R)$ with greater precision that could in turn constrain the search for dark matter in colliders.
\end{abstract}

Keywords: Dark Matter; Scalaron; Chameleon mechanism.

\section{Introduction}

The explanation of dark matter and the late-time accelerated expansion of the universe are the two major challenges of the present day cosmology. There are several observational evidences such as Supernovae Ia, Baryon Acoustic Oscillation (BAO), Cosmic Microwave Background anisotropies, weak gravitational lensing etc. 1,2,3,4 which indicate that the present universe is in the phase of accelerated expansion. There are several approaches to explain the late-time accelerated expansion. The most suitable model to explain such acceleration is the Lambda Cold Dark Matter $(\Lambda \mathrm{CDM})$ model, where $\Lambda$ is the well known cosmological constant ${ }^{5,6}$. However, this constant faces two serious problems- $(i)$ coincidence problem, and ( $i i)$ fine tuning problem. In addition to it, the fundamental nature of the cosmological constant is

This is an Open Access article published by World Scientific Publishing Company. It is distributed under the terms of the Creative Commons Attribution 4.0 (CC-BY) License. Further distribution of this work is permitted, provided the original work is properly cited. 
unknown. Quite often, an explicit matter component with strange characteristics is also introduced to explain the cosmic acceleration. It is known as the dark energy $7,8,9,10$. There exist another approach called modified gravity, wherein the late-time accelerated expansion is explained without using any explicit dark energy component $11,12,13,14,15$. The modified gravity models include $f(R)$ theory, Braneworld models, Gauss-Bonnet dark energy models etc., out of which $f(R)$ theory is the simplest modified gravity models, in which the Ricci scalar $R$ of Hilbert-Einstein action is generalised into a function $f(R)$ of $R$. Besides the existence of late-time accelerated expansion of the universe, the observations of rotation curves of galaxies ${ }^{16,17}$ and gravitational lensing indicate the presence of new matter often known as dark matter. This matter does not interact with the electromagnetic radiation, even though it has the gravitational interaction with the normal matter and radiation. The fundamental nature of the dark matter is mysterious. There are some famous candidates of dark matter like weakly interacting massive particles (WIMPs) ${ }^{18}$. Several approaches exist to explain the dark matter in modified gravity theories ${ }^{19,20,21}$.

Here, we will discuss the dark matter problem in $f(R)$ gravity. There are three approaches to derive the field equations in $f(R)$ models - $(i)$ metric formalism $(i i)$ Palatini formalism and (iii) metric-affine formalism. Under the conformal transformation of spacetime metric from Jordan frame to Einstein frame, an scalar field emerges. An arrow of time is shown by using this scalar field ${ }^{22}$ and other approach for $f(R)=\alpha R^{2}$ model ${ }^{23}$. In $f(R)$ gravity dark matter is explained without considering any extra matter component. Here, we use $f(R)=R^{1+\delta}$, where $\delta<<1$, to explain the dark matter phenomena.

The present paper is organised in four sections. Section (2) contains the phase space analysis of the $f(R)$ gravity and conformal transformation. In section (3) dark matter and chameleon mechanism in $f(R)$ gravity are discussed. We conclude and discuss our results in section (4).

\section{Phase space analysis of $f(R)$ gravity and conformal transformation}

We consider the 4-dimensional action of the $f(R)$ gravity model given by some general function $f(R)$ of the Ricci scalar

$$
\mathcal{A}=\frac{1}{2 \kappa^{2}} \int d^{4} x \sqrt{-g} f(R)+\mathcal{A}_{m}\left(g_{\mu \nu}, \Psi_{m}\right)
$$

where $\kappa^{2}=8 \pi G$ and $\mathcal{A}_{m}$ is the action of the matter part with matter field $\Psi_{m}$.

Here, we use the metric formalism in which connections $\Gamma_{\beta \gamma}^{\alpha}$ are defined in terms of the metric tensor $g_{\mu \nu}$. Varying the action (1) w.r.t. $g_{\mu \nu}$, we get the field equations given by

$$
\begin{array}{r}
F(R) R_{\mu \nu}-\frac{1}{2} f(R) g_{\mu \nu} \\
-\nabla_{\mu} \nabla \nu F(R)+g_{\mu \nu} \square F(R)=\kappa^{2} T_{\mu \nu}
\end{array}
$$


where $F(R) \equiv \frac{\partial f}{\partial R}$ and $T_{\mu \nu}$ is the energy-momentum tensor for matter. The trace of field equation (2) is given by

$$
3 \square F(R)+F(R) R-2 f(R)=\kappa^{2} T,
$$

where $T=-\rho+3 p$. Here $\rho$ and $p$ are the energy density and the pressure of matter respectively. Trace of the field equations shows that Ricci scalar is dynamical if $F(R) \neq R$.

In the phase space analysis of $f(R)$ models, we define four (dimensionless) variables as:

$$
x_{1} \equiv-\frac{\dot{F}}{F H} ; x_{2} \equiv-\frac{f}{6 F H^{2}} ; x_{3} \equiv \frac{R}{6 H^{2}} ; x_{4} \equiv \frac{\kappa^{2} \rho_{r}}{3 F H^{2}}
$$

where $\dot{F}$ is the derivative of $F(R)$ w.r.t. time, $H \equiv \frac{\dot{a}}{a}$ is the Hubble parameter and $\rho_{r}$ is the energy density of radiation. Varying these variables w.r.t. $N \equiv \ln a(t)$, and equating to zero, we get the fixed points of the dynamical system. There are eight fixed points and stability of each point is determined by taking the perturbation about each point. We take different points corresponding to different phases of the universe. Using these fixed points and some basics definitions, we get the form of $f(R)$ and other parameters for different phases of the universe. The form of $f(R)$ for radiation dominated phase is $\alpha R$ and that for the matter dominated and late-time accelerated expansion dominated phases are $\beta R$ and $\gamma R^{2}$, respectively. Similarly other parameters like Hubble parameter $H$ and Ricci scalar can also be calculated.

We rewrite the action (1) in the form

$$
\mathcal{A}=\int \sqrt{-g}\left(\frac{1}{2 \kappa^{2}} F(R) R-U\right) d^{4} x+\mathcal{A}_{m},
$$

where

$$
U=\frac{F(R) R-f(R)}{2 \kappa^{2}}
$$

For the solution of dark matter problem we consider the Einstein frame. It is possible to derive an action in the Einstein frame under the conformal transformation

$$
\tilde{g}_{\mu \nu}=\Omega^{2} g_{\mu \nu},
$$

where $\Omega^{2}$ is the conformal factor and a tilde denotes the quantities pertaining to the Einstein frame. The corresponding Ricci scalars in the two frames are related as

$$
R=\Omega^{2}\left(\tilde{R}+6 \tilde{\square} \omega-6 \tilde{g}^{\mu \nu} \partial_{\mu} \omega \partial_{\nu} \omega\right)
$$

where

$$
\omega \equiv \ln \Omega, \partial_{\mu} \omega \equiv \frac{\partial \omega}{\partial \tilde{x}^{\mu}}, \tilde{\square} \omega \equiv \frac{1}{\sqrt{-\tilde{g}}} \partial_{\mu}\left(\sqrt{-\tilde{g}} \tilde{g}^{\mu \nu} \partial_{\nu} \omega\right)
$$


Thus, the action (5) under the conformal transformation is transformed as

$$
\begin{array}{r}
\mathcal{A}=\int d^{4} x \sqrt{-\tilde{g}}\left[\frac{1}{2 \kappa^{2}} F \Omega^{-2}\left(\tilde{R}+6 \tilde{\square} \omega-6 \tilde{g}^{\mu \nu} \partial_{\mu} \omega \partial_{\nu} \omega\right)-\Omega^{-4} U\right] \\
+\mathcal{A}_{m}
\end{array}
$$

The linear action in $\tilde{R}$ can be written by choosing

$$
\Omega^{2}=F(R)
$$

We consider a new scalar field $\phi$ defined by

$$
\kappa \phi \equiv \sqrt{\frac{3}{2}} \ln F(R) .
$$

Using these relations the action in Einstein frame is found as

$$
\mathcal{A}=\int d^{4} x \sqrt{-\tilde{g}}\left[\frac{1}{2 \kappa^{2}} \tilde{R}-\frac{1}{2} \tilde{g}^{\mu \nu} \partial_{\mu} \phi \partial_{\nu} \phi-V(\phi)\right]+\mathcal{A}_{m}
$$

where

$$
V(\phi)=\frac{U}{(F(R))^{2}}=\frac{F(R) R-f}{2 \kappa^{2}(F(R))^{2}}
$$

\section{Dark matter in $f(R)$ models}

From the constant tangential velocity condition on the motion of a test particle in the stable orbits of the galaxies, the form of $f(R)$ is given by $f(R)=R^{1+\delta}$, where $\delta<<1$. Therefore to solve the problem of dark matter, only very small deviation from general relativistic theory is required. There is another approach to overcome the dark matter issue in $f(R)$ gravity. In this approach it is shown that the dark matter can be scalar field particle "scalaron". In the Einstein frame the scalar field $\phi$ is coupled with non-relativistic matter. This coupling has the relation

$$
\Omega^{2}=F(R)=e^{-2 Q \kappa \phi},
$$

where $Q$ is the strength of the coupling. Now, from equation (12) and equation (15), $Q$ is given by

$$
Q=-\frac{1}{\sqrt{6}}
$$

Let us consider the dark matter problem for $f(R)$ model given by

$$
f(R)=R^{1+\delta}
$$

Differentiating equation (16) with respect to $R$, we get

$$
F(R)=(1+\delta) R^{\delta}
$$


From equations (15) and (18) for $Q=-\frac{1}{\sqrt{6}}$, Ricci scalar $R$ in terms of scalar field $\phi$ is given as:

$$
R=\left[\frac{e^{\sqrt{2 / 3} \kappa \phi}}{1+\delta}\right]^{\frac{1}{\delta}}
$$

The effective potential of the scalaron for non-relativistic matter is given by

$$
V_{e f f}=\frac{\delta}{2 \kappa^{2}(1+\delta)^{2}}\left[\frac{e^{\sqrt{\frac{2}{3}} \kappa \phi}}{(1+\delta)}\right]^{\frac{1-\delta}{\delta}}+\frac{1}{4} \rho e^{\frac{-4 \kappa \phi}{\sqrt{6}}}
$$

To find the value of $\phi$ at which $V_{e f f}$ is minimum, we calculate $\frac{d V_{e f f}}{d \phi}$ as

$$
V_{e f f}^{\prime}=\frac{1}{\sqrt{6} \kappa} \frac{(1-\delta)}{(1+\delta)^{\frac{1+\delta}{\delta}}}\left[e^{\sqrt{\frac{2}{3}} \kappa \phi}\right]^{\frac{(1-\delta)}{\delta}}-\frac{\kappa}{\sqrt{6}} \rho e^{\frac{-4 \kappa \phi}{\sqrt{6}}}
$$

Solving $V_{e f f}^{\prime}=0$, we get the minimum value of the scalar $\phi$ given by,

$$
\phi_{\text {min }}=\sqrt{\frac{3}{2}} \frac{1}{\kappa} \ln \left[(1+\delta)\left(\frac{\kappa^{2} \rho}{1-\delta}\right)^{\frac{\delta}{1+\delta}}\right]
$$

For the calculation of mass of the scalar field, we have

$$
V^{\prime \prime}(\phi)=\frac{1}{3} \frac{(1-\delta)^{2}}{\delta(1+\delta)^{\frac{1+\delta}{\delta}}}\left[e^{\sqrt{\frac{2}{3}} \kappa \phi}\right]^{\frac{(1-2 \delta)}{\delta}}+\frac{2 \kappa}{3} \rho e^{\frac{-4 \kappa \phi}{\sqrt{6}}}
$$

The mass of the scalaron $\left(m_{\phi}\right)$ is given by

$$
m_{\phi}^{2}=\frac{(1-\delta)^{\frac{1}{1+\delta}}}{3 \delta(1+\delta)^{3}} \rho^{\frac{1-2 \delta}{1+\delta}}+\frac{2}{3} \frac{(1-\delta)^{\frac{2 \delta}{1+\delta}}}{(1+\delta)^{2}} \rho^{\frac{1-\delta}{1+\delta}}
$$

The mass of the scalar field depends upon the energy density of the matter. The chameleon mechanism works in the high energy density regions and from the equation (24), it is found that the mass of the scalaron increases with the energy density. The scalar field is coupled with the standard matter particles and the mass of the scalaron is large in high curvature regime. Therefore, the motion of the scalar field is diminished and the local gravity constraints are satisfied. Thus the behavior of scalar field changes with the length scale due to chameleon mechanism. These properties of scalaron show that the it could be the dark matter.

\section{Conclusion}

We investigated here the possibility of explaining the dark matter problem in $f(R)$ gravity. It is shown that the dark matter can be found without adding new matter component to the particle contents of the standard model. For $f(R)=R^{(1+\delta)}$ model, the mass of the scalaron almost linearly depends upon the energy-density of the standard matter particles. Therefore, it doesn't change very much in large curvature regimes in comparison to the other models (like the Starobinsky model) 
in which there is a power law dependence. This behavior is suitable for the dark matter phenomena. We did not find any condition on the amount of the dark matter content and the scale dependence of the $\delta$, i.e., the deviation from the Einstein's general relativistic theory. We will deal with these problems in our future work. It is also possible to work on the unified theory of dark matter and dark energy in this approach. This important work distinguishes the role of dark matter in cosmological models, namely, $f(R)$ gravity model and the standard $\Lambda$ CDM model. Here we have used the form of $f(R)=R^{(1+\delta)}$, where $\delta<<1$ from the constraints on the tangential velocity of a test particle in stable orbits. But, in this approach we did not find any constraint on $\delta$. It is possible to get some condition on the $\delta$ from observations. The observations in the accelerated phase of the universe can make such distinction and further would constrain the dark matter searches in colliders like LHC.

\section{Acknowledgments}

Authors thank the Inter University Centre for Astronomy and Astrophysics (IUCAA), Pune for the local hospitality and computational facilities under the associateship programme, where a part of the present work was completed.

\section{References}

1. A. G. Riess et al., (Supernova Search Team Collaboration), Astron. J. 116, 1009 (1998).

2. B. Schmidt et al., Astrophys. J. 507, 46 (1998).

3. S. Perlmutter et al., Astrophys. J. 517, 565 (1999).

4. D. N. Spergel et al., Astrophys. J. Suppl. 148, 97 (2003).

5. S. M. Carroll, Living Rev. Relativ.(2001)4:1.

6. P. J. E. Peebles and Bharat Ratra, Rev. Mod. Phys. 75, 559-606 (2003).

7. R. R. Caldwell, R. Dave, and P. J. Steinhardt, Phys. Rev. Lett. 80, 1582 (1998).

8. S. Capozziello, Int. J. Mod. Phys. D 11, 483 (2002)

9. T. Chiba, T. Okabe, and M. Yamaguchi, Phys. Rev. D 62, 023511 (2000).

10. R. R. Caldwell, Phys. Lett. B 545, 23 (2002).

11. M. Kunz and D. Sapone, Phys. Rev. Lett. 98, 121301 (2007).

12. L. Amendola, R. Gannouji, D. Polarski and S. Tsujikawa, Phys. Rev. D 75, 083504 (2007).

13. V. Sahni and Y. Shtanov, JCAP 0311, 014 (2003).

14. A. A. Starobinski, Phys. Lett. B 91, 99(1980).

15. S. Nojiri and S. D. Odintsov, Phys. Rept. 505, 59 (2011).

16. V. C. Rubin, W. K. Ford and N. Thonnard, Astrophys. J. 238, 471 (1980).

17. A. Borriello and P. Salucci, Mon. Not. R. Astron. Soc. 323, 285 (2001).

18. G. Jungman, M. Kamionkowski and K. Griest, Phys. Rep. 267, 195 (1996).

19. C. G. Boehmer, T. Harko and F. S. N. Lobo, Astropart. Phys. 29, 386 (2008).

20. T. Katsuragawa and S. Matsuzaki, Phys. Rev. D 95, 044040 (2017).

21. R. Zaregonbadi, M. Farhoudi and N. Riazi, Phys. Rev. D 94, 0840052 (2016).

22. M. M. Verma and B. K. Yadav, Int. J. Mod. Phys. D 27, 1850002 (2018).

23. B. K. Yadav and M. M. Verma, Int. J. Mod. Phys. D 26, 1750183 (2017). 